EPJ Web of Conferences 71, 00102 (2014)

DOI: 10.1051/epjconf/20147100102

(C) Owned by the authors, published by EDP Sciences, 2014

\title{
Study of the SM Higgs boson in the ZZ channel at CMS
}

\author{
Giacomo Ortona ${ }^{1, a}$, on behalf of the CMS collaboration \\ ${ }^{1}$ Università degli Studi di Torino
}

\begin{abstract}
On July $4^{\text {th }}, 2012$ the ATLAS and CMS collaborations announced the discovery of a new Higgs-like resonance at a mass of about $126 \mathrm{GeV}$. To determine if the observed resonance is compatible with a SM Higgs boson, the resonance characteristics (mass, couplings, spin) must be measured with high precision. The $Z Z$ channel $\left(H \rightarrow Z Z^{*} \rightarrow 4 l, l=e, \mu\right)$ is one of the most promising channels for these measurements. The CMS analysis $H \rightarrow Z Z^{*} \rightarrow 4 l$ is presented, covering its most important aspects: lepton identification, isolation variables, kinematics, event selection, and background control. The talk presents the most recent CMS results on the search for the standard model Higgs boson in the $Z Z$ channel, using the full dataset recorded at the LHC from $p p$ collisions at centre of mass energies of 7 and $8 \mathrm{TeV}$. The properties of the new boson, such as the mass and spin, are discussed in detail.
\end{abstract}

\section{Introduction}

The standard model of electroweak interactions (SM) relies on the existence of a scalar particle (the Higgs boson) associated with the field responsible for the spontaneous electroweak symmetry breaking. Since the announcement of the discovery of a new particle made on July $4^{\text {th }}, 2012$ by the CMS [1] and ATLAS [2] collaborations much effort was dedicated by both collaborations to determine the compatibility of this new particle with the long sought standard model Higgs boson using the full set of data collected in 2011 and 2012.

In CMS, the $H \rightarrow Z Z^{*} \rightarrow 4 l$, with $l=e, \mu$ is a particularly good channel not only to search for the presence of a new particle, but also to study its characteristics. Despite having a rather small branching fraction when compared to other production mechanism [3], the $H \rightarrow Z Z^{*} \rightarrow 4 l$ channel can profit from a very clear experimental signature: a peak in the invariant mass of two pairs of opposite sign same flavour charged leptons over a small and flat background. The signal over background ratio in this channel is about a factor 2 for a Higgs boson with a mass of $126 \mathrm{GeV}$. This makes the $H \rightarrow$ $Z Z^{*} \rightarrow 4 l$ channel the most sensitive channel for the search of a low mass Higgs boson available in CMS. Another important characteristic of this decay channel is that the decay topology is fully reconstructed. The precise knowledge of the mass and of the angular distributions of all the final state particles can be used to reach high sensitivity when studying the mass of the reconstructed resonance, to improve the discrimination power between signal and background, and to study the spin and the parity of the new particle.

\footnotetext{
ae-mail: ortona@to.infn.it
} 


\section{The CMS experiment}

The central feature of the Compact Muon Solenoid (CMS) apparatus is a superconducting solenoid of $6 \mathrm{~m}$ internal diameter, providing a magnetic field of 3.8 T. Within the superconducting solenoid volume are a silicon pixel and strip tracker, a lead tungstate crystal electromagnetic calorimeter (ECAL), and a brass/scintillator hadron calorimeter (HCAL). Muons are measured in gas-ionisation detectors embedded in the steel return yoke outside the solenoid. Extensive forward calorimetry complements the coverage provided by the barrel and endcap detectors.

The particle-flow event reconstruction consists in reconstructing and identifying each single particle with an optimised combination of all subdetector information. The energy of electrons is determined from a combination of the track momentum at the main interaction vertex, the corresponding ECAL cluster energy, and the energy sum of all bremsstrahlung photons attached to the track. The energy of muons is obtained from the corresponding track momentum.

The mass resolution for $Z \rightarrow e^{+}+e^{-}$decays when both electrons are in the ECAL barrel is $1.6 \%$, and is $2.6 \%$ when both electrons are in the endcaps. The electron energies are measured by the ECAL and their directions are measured by the tracker [4].

Muons are measured in the pseudorapidity range $|\eta|<2.4$, with detection planes made using three technologies: drift tubes, cathode strip chambers, and resistive plate chambers. Matching muons to tracks measured in the silicon tracker results in a transverse momentum resolution between 1 and $5 \%$, for $p_{\mathrm{T}}$ values up to $1 \mathrm{TeV}$

A more detailed description can be found in [4].

\section{Analysis Description}

\subsection{Event selection}

The results presented in these proceedings have been obtained from a $5.1 \mathrm{fb}^{-1}$ dataset collected at the LHC at an energy of $\sqrt{s}=7 \mathrm{TeV}$ during 2011 and a $19.6 \mathrm{fb}^{-1} \sqrt{s}=8 \mathrm{TeV}$ dataset collected in 2012 . For passing the trigger requirements events must have at least two leptons with a minimal transverse momentum of 17 (8) GeV for the leading (subleading) lepton. To increase the efficiency in the case of the $4 \mathrm{e}$ final state, also events with three electrons passing transverse momentum thresholds of 15,8 , and $5 \mathrm{GeV}$ are selected. The trigger efficiency for events within the geometrical acceptance is greater than $98 \%$ for a Higgs boson signal with mass larger than $110 \mathrm{GeV}$.

Pairs of same flavour / opposite sign leptons are combined to build $Z Z$ pairs. Muons (electrons) are required to have $p_{T}^{\mu}>5 \mathrm{GeV}\left(p_{T}^{e}>7 \mathrm{GeV}\right)$. To further reject background, at least one of the 4 leptons must have $p_{T}>20 \mathrm{GeV}$ and a second one $p_{T}>10 \mathrm{GeV}$. We assign the label of $Z_{1}$ to the reconstructed $Z$ closest to the nominal $Z$ mass, and $Z_{2}$ to the other. For the candidate $Z Z$ pair to be considered, we ask that $40<m_{Z_{1}}<120 \mathrm{GeV}$ and $12<m_{Z_{2}}<120 \mathrm{GeV}$. As further requirements we also select only $Z Z$ pairs where the invariant mass of the 4-lepton system is larger than $100 \mathrm{GeV}$ and in which any opposite charge pair mass is larger than $4 \mathrm{GeV}$. Leptons can lose energy emitting final state radiation (FSR). Reconstructed isolated photons with $p_{T}^{\gamma}>2 \mathrm{GeV}$ found in a cone of size $\Delta R<0.07$ from one of the selected lepton, or with $p_{T}^{\gamma}>4 \mathrm{GeV}$ and found isolated from charged particles and energy deposits within $0.07<\Delta R<0.5$ from a selected lepton candidate are attached to the selected lepton to recover for FSR. Further details on the selection can be found in [5]. The overall selection efficiency is $31 \%, 42 \%$, and $59 \%$ for the $4 e, 2 e 2 \mu$, and $4 \mu$ final states respectively.

The background sources have an irreducible four-lepton contribution from $Z Z^{*}$ or $Z \gamma^{*}$ production via $q \bar{q}$ annihilation or $g g$ fusion, and a reducible contribution. The reducible contribution arise from $Z b \bar{b}$ and $t \bar{t}$ processes with two prompt leptons in the final state and two more secondary leptons 


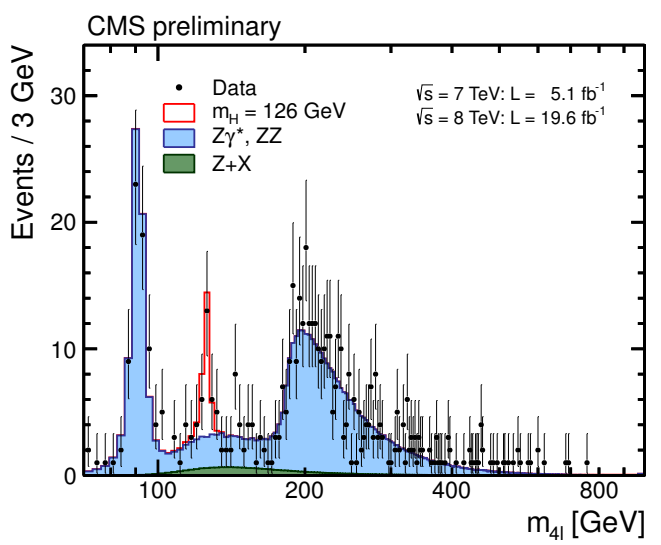

Figure 1: Distribution of the four-lepton reconstructed mass from the full dataset collected at $\sqrt{s}=7$ and $\sqrt{s}=8 \mathrm{TeV}$. Points represent the data, shaded histograms represent the background and the unshaded histogram the signal expectation for a Higgs boson with $m_{H}=126 \mathrm{GeV}$. No observed event is present above $m_{4 l}>800 \mathrm{GeV}$.

coming from $b$ jets. Additional instrumental background arises from jets misidentified as leptons in $Z+$ jets, $Z+\gamma+$ jets, and $W Z+$ jets events. The irreducible contribution is evaluated using Monte Carlo simulation. Data-driven techniques, based on the probability for a reconstructed secondary lepton to pass the selection requirements (tag and probe) are used to evaluate the contribution from reducible and instrumental background.

The 4-lepton invariant mass distribution obtained combining $4 e, 2 e 2 \mu$, and $4 \mu$ final states is shown in figure 1, together with the expectation from SM background processes. The observed distribution is in good agreement with the expectation. The resonant peak for the $Z \rightarrow 4 l$ process at $m_{4 l}=m_{Z}$ is observed with the expected normalisation and shape and provides a basic test of the accuracy of the Monte Carlo used to simulate the background processes. A clear peak is visible at $m_{4 l}=126 \mathrm{GeV}$, thus confirming the presence of the Higgs boson already announced in [1].

\subsection{Event categorization}

For each event, 3 different variables are built in order to perform the statistical analysis. Alongside the 4-lepton invariant mass, a second variable, the kinematic discriminant $K_{D}=\frac{P_{0^{+}}^{\mathrm{kin}}}{P_{0^{+}}^{\mathrm{kik}}+P_{\mathrm{bkg}}^{\mathrm{kin}}}=$ $\left(1+\frac{P_{\mathrm{bkg}}^{\mathrm{kin}}\left(m_{1}, m_{2}, \Omega \mid m_{4 l}\right)}{P_{0^{+}}^{\mathrm{kin}}\left(m_{1}, m_{2}, \Omega \mid m_{4 l}\right)}\right)^{-1}$ is built from a Matrix Element Likelihood Analysis (MELA) based on the probability ratio of the signal and background hypotheses given the five angles and the two $\left(m_{Z_{1}} \cdot m_{Z_{2}}\right)$ masses that completely define the event kinematics [6]. The same approach can be used to build a discriminant to separate between different signal hypotheses instead of between signal and background. Such a discriminant can be used to asses the spin and the parity of the observed Higgs boson.

To improve the sensitivity of the analysis to the production mechanism, the event sample is split into two categories according to their jet multiplicity. Events in the $0 / 1$ jet category (category I) contain less than 2 jets, while events in the Dijet category (category II) contain at least 2 jets with minimum transverse momentum of $30 \mathrm{GeV}$. In each category a third discriminating variable is used 


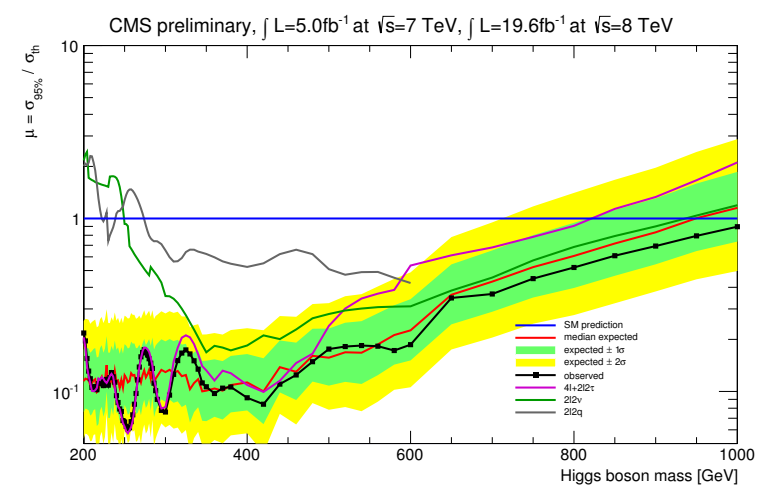

Figure 2: Combined (black points) and single-channel (purple line: $4 l$, green line: $2 l 2 \bar{v}$, grey line: $2 l 2 q$ ) observed and expected (red line) 95\% CL upper limit on the ratio of the production cross section to the SM expectation. The expected $68 \%$ and $95 \%$ CL ranges of expectation for the background-only model are also shown with green and yellow bands, respectively.

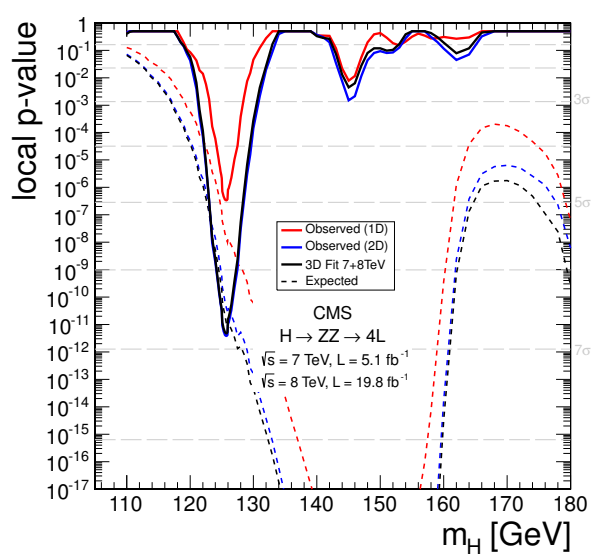

Figure 3: Significance of the local excess with respect to the SM background expectation as a function of the Higgs boson mass in the mass range 110-1000 GeV. Results are shown for the 1D fit (red), 2D fit (blue), and the nominal 3D fit (black). Dashed lines represent the SM expectation for a given mass Higgs boson.

together with the 4-lepton mass and $K_{D}$ when performing the fit of the results. The variable associated to category I is $p_{T}^{\text {jet }} / m_{4 l}$, while a Fisher discriminant $D_{\text {jet }}=\alpha \Delta \eta_{j j}+\beta m_{j j}$ is used for the Dijet category. This discriminant is tuned in order to separate vector boson fusion (VBF) from gluon fusion processes. In the $0 / 1$ jet category about $5 \%$ of the signal events are expected to come from the VBF production mechanism, while this number increases to $20 \%$ in category II.

\section{Results}

In the $H \rightarrow Z Z^{*} \rightarrow 4 l$ analysis an unbinned maximum likelihood fit performed to the selected events is used to measure the various Higgs properties under study, such as signal and background yields, the mass of the resonance, and the spin-parity quantum numbers. Different multi-dimensional models are used depending on the specific properties of interest, as will be detailed in this section.

\subsection{Searches, observation and mass}

To measure the compatibility of the observed data distribution with the background-only hypothesis, the exclusion limits, and the signal strength $\mu=\sigma / \sigma_{\mathrm{SM}}$ defined as the measured cross section times branching ratio in $Z Z$ divided by the SM expectation, a 3-dimensional likelihood function is defined as follows in the 0/1 jet category: $\mathscr{L}\left(m_{4 l}, K_{D}, p_{T}\right)=P\left(p_{T} / m_{4 l} \mid m_{4 l}\right) \times P\left(K_{D} \mid m_{4 l}\right) \times P\left(m_{4 l} \mid m_{H}\right)$ and in the Dijet category: $\mathscr{L}\left(m_{4 l}, K_{D}, p_{T}\right)=P\left(D_{\text {jet }} \mid m_{4 l}\right) \times P\left(K_{D} \mid m_{4 l}\right) \times P\left(m_{4 l} \mid m_{H}\right)$. To test the significance of the observed signal and to establish exclusion limits, the selected events are split into twelve subcategories based on the three final states, the two data-taking periods ( 7 and $8 \mathrm{TeV}$ ), and the two 
jet categories. These events are examined for 187 hypothetical SM-like Higgs boson masses in a range between $110 \mathrm{GeV}$ and $1000 \mathrm{GeV}$. The significance is computed and exclusion limits are set following the statistical approach discussed in Ref. [7]. Limits are reported according to the modified frequentist construction CLs. The limit range at high mass can be extended including $Z$ decading into $\tau$ to improve the high mass sensitivity. With this addition the $H \rightarrow Z Z^{*} \rightarrow 4 l$ analysis is capable to exclude the presence of the Higgs boson from a mass of $130 \mathrm{GeV}$ up to $827 \mathrm{GeV}$ (expected range for SM Higgs is $113.5-778$ ). The inclusion of other final states such as $2 l 2 \bar{v}$ and $2 l 2 q$ allow to further extend this limit up to $1 \mathrm{TeV}$, as reported in figure 2. As shown in figure 3 the minimum of the local p-value is reached for $m_{H}=125.7 \mathrm{GeV}$ and the signal excess is measured to be 6.7 Gaussian $\sigma$, to be compared with an expected value of 7.2 $\sigma$. When neglecting the jet categorization the $2 \mathrm{D}$ observed pvalue is equal to $6.6 \sigma$ ( 6.9 expected). At the same mass value the signal strength is $\mu=0.91_{-0.24}^{+0.30}$. The signal strengths in the two jet categories are measured to be $\mu_{\text {Dijet }}=1.22_{-0.57}^{+0.84}$ and $\mu_{\text {untag }}=0.85_{-0.26}^{+0.32}$ in category I and II respectively. The value of the signal strength modifier scale factors for the vector boson $(\mathrm{VBF}, \mathrm{VH})$ and fermion $(\mathrm{ggH}, t \bar{t} H)$ contributions to the total cross section are $\mu_{v}=1.0_{-2.3}^{+2.4}$ and $\mu_{f}=0.9_{-0.4}^{+0.5}$. All signal strengths are compatible with the SM expectations.

To measure the mass of the observed Higgs boson, the per-event mass errors of each candidate are incorporated in the likelihood fit together with the $K_{D}$ and the 4-lepton mass. The dominant source of systematic uncertainty for this measurement comes from the effect on the mass scale of the lepton momentum scale which is known with limited precision. Figure 4 show the 3D likelihood scan for the three final states separately. The signal strength is left free to float in the fit. It is possible to observe that the three channels lead to compatible result among them and with the combined fit, that gives a best estimate for the Higgs boson mass of $m_{H}=125.8 \pm 0.5$ (stat.) \pm 0.2 (syst.) $\mathrm{GeV}$.

\subsection{Spin-parity measurement}

In the $H \rightarrow Z Z^{*} \rightarrow 4 l$ the $\mathrm{SM}$ has been tested against six alternative spin-parity models $\left(D_{0^{-}}, D_{0_{h}^{+}}, D_{1^{-}}, D_{1^{+}}, D_{2_{m}^{+}}^{g g h}, D_{2_{m}^{+}}^{q q}\right)$. For each of the tested spin-parity models, a new kinematic discriminant defined as $D_{J^{P}}=\frac{P_{\mathrm{SM}}}{c^{\prime} \times P_{\mathrm{SM}}+P_{J} P}=\left[1+\frac{c^{\prime} \times P_{J P}^{\mathrm{ang}}}{P_{\mathrm{SM}}^{\mathrm{ang}}}\right]^{-1}$ is built using the MELA approach in order to discriminate between the SM and the alternative hypothesis. The $c^{\prime}$ factor is tuned in order to adjust the relative normalisation of the probabilities. Monte Carlo simulations are used to generate 2D templates of $D_{J^{p}}$ versus $D_{\mathrm{bkg}}$ and the likelihood distributions for the signal and the alternative hypothesis under test. The test statistic $q=-2 \ln \frac{L_{J^{P}}}{L_{0^{+}}}$distribution is generated using Monte Carlo samples for the $\mathrm{SM}$ and the other six models. An example of such a distribution for the $J^{P}=0^{-}$hypothesis, compared to the SM one, is shown in figure 5. A CLs criterion is defined as the ratio of the probabilities to observe a value of the test statistics q equal or larger than the one in the data under the SM or the $J^{P}$ hypotheses. Using this criterion, the SM is clearly favoured against all other tested hypotheses. The results for each of the six tests are reported in table 1.

It is possible to write the Higgs decay amplitude as:

$$
A(H \rightarrow Z Z)=v^{-1} \epsilon_{1}^{*} \epsilon_{2}^{*}\left(a_{1} g_{\mu v} m_{Z}^{2}+a_{2} q_{\mu} q_{v}+a_{3} \epsilon_{\mu \nu \alpha \beta} q_{1}^{\alpha} q_{2}^{\beta}\right)=A_{1}+\not A_{2}+A_{3} .
$$

Considering the $A_{2}$ term as negligible, the SM amplitude is dominated by the $A_{1}$ term while the pseudoscalar $0^{-}$model is dominated by $A_{3}$. Neglecting interference effects, which should be very small, it is possible to define the fraction of CP-odd contributions to the observed cross section defined as $f_{a 3}=\left|A_{3}\right|^{2} /\left(\left|A_{1}\right|^{2}+\left|A_{3}\right|^{2}\right)$. The shape of the $D_{0^{-}}$discriminant distribution is highly sensitive to the value of $f_{a 3}$. Such discriminant is therefore an optimal tool to measure the value of this contribution. The measured value is $f_{a 3}=0.00_{-0.00}^{+0.23}$, compatible with the SM prediction (0). 


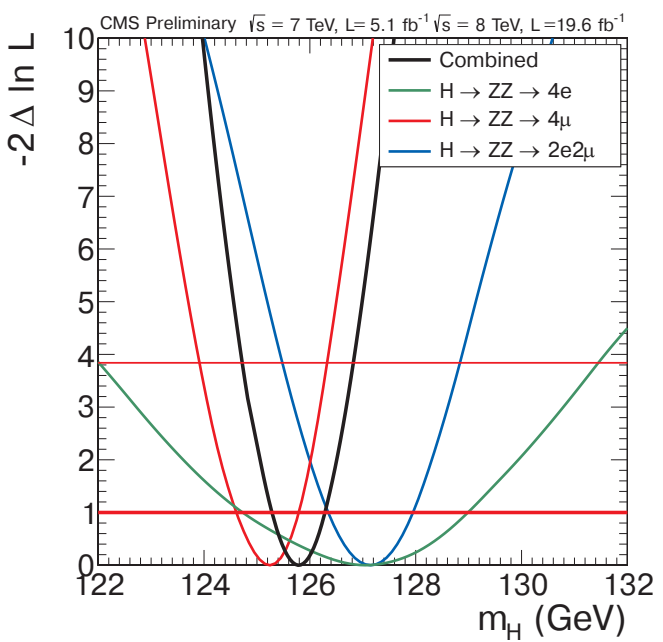

Figure 4: Scan of the negative 3D log-likelihood $-2 \Delta \ln \mathscr{L}$ versus tested SM Higgs boson mass, for each of the three channels separately and the combination of the three. Dashed line represents the scan including only statistical uncertainties.

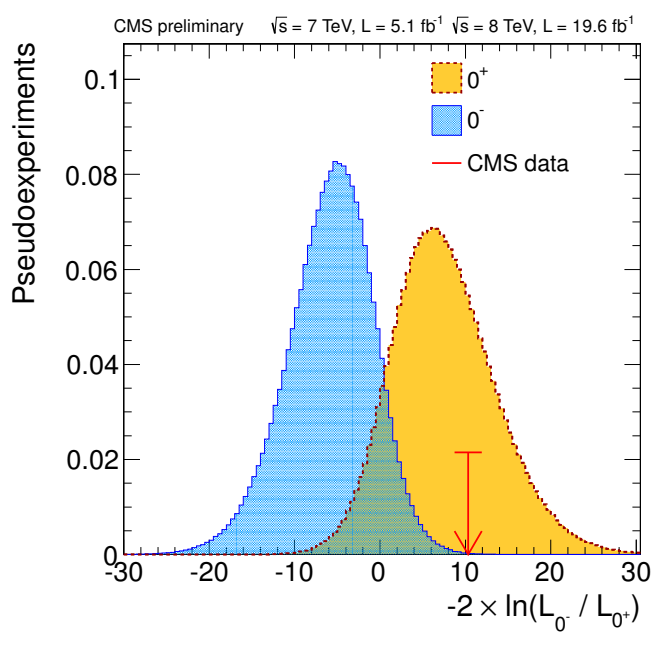

Figure 5: Distribution of the test-statistic $q=$ $-2 \ln \frac{L_{0^{-}}}{L_{0^{+}}}$of the pseudoscalar boson hypothesis tested against the SM Higgs boson hypothesis. Distributions for the SM Higgs boson are represented by the yellow histogram and for the alternative hypotheses by the blue histogram. The arrow indicates the observed value.

Table 1: List of models used in analysis of spin-parity hypotheses corresponding to the pure states of the type noted. Events for the expected separation are generated with SM expectation for the signal yield $(\mu=1)$. The observed separation quotes consistency of the observation with the $0^{+}$model or $J^{P}$ model, and corresponds to a scenario when the signal strength is pre-determined from the fit to data. The last column quotes CLs criterion for the $J^{P}$ model as described in the text.

\begin{tabular}{|c|c|c|c|c|c|}
\hline$J^{P}$ model & $J^{P}$ production & expect $(\mu=1)$ & obs. $0^{+}$ & obs. $J^{P}$ & CL $_{s}$ \\
\hline $0^{-}$ & $g g \rightarrow X$ & $2.6 \sigma(2.8 \sigma)$ & $0.5 \sigma$ & $3.3 \sigma$ & $0.16 \%$ \\
$0_{h}^{+}$ & $g g \rightarrow X$ & $1.7 \sigma(1.8 \sigma)$ & $0.0 \sigma$ & $1.7 \sigma$ & $8.1 \%$ \\
$2^{+}$ & $g g \rightarrow X$ & $1.8 \sigma(1.9 \sigma)$ & $0.8 \sigma$ & $2.7 \sigma$ & $1.5 \%$ \\
$2^{+}$ & $q \bar{q} \rightarrow X$ & $1.7 \sigma(1.9 \sigma)$ & $1.8 \sigma$ & $4.0 \sigma$ & $<0.1 \%$ \\
$1^{-}$ & $q \bar{q} \rightarrow X$ & $2.8 \sigma(3.1 \sigma)$ & $1.4 \sigma$ & $>4.0 \sigma$ & $<0.1 \%$ \\
$1^{+}$ & $q \bar{q} \rightarrow X$ & $2.3 \sigma(2.6 \sigma)$ & $1.7 \sigma$ & $>4.0 \sigma$ & $<0.1 \%$ \\
\hline
\end{tabular}

\section{Conclusions}

The analysis of the Higgs boson decaying to a pair of $Z$ bosons has been presented, focusing on the results with 4 leptons in the final state. A clear peak is visible above the background at a mass around $126 \mathrm{GeV}$. The significance of the observed peak is $6.7 \sigma$, above the discovery threshold in this channel alone. The ratio between the measured cross section times branching ratio and the SM expectation is $\mu=0.91_{-0.24}^{+0.30}$, compatible with the SM. The vector boson and fermionic contribution to the observed 


\section{ICNFP 2013}

cross section, obtained dividing the data sample in jet categories, are also compatible with the SM $\left(\mu_{v}=1.0_{-2.3}^{+2.4}\right.$ and $\left.\mu_{f}=0.9_{-0.4}^{+0.5}\right)$. The presence of a SM Higgs boson at a different mass is excluded up to $1 \mathrm{TeV}$. The spin-parity analysis strongly disfavour the tested pure alternative models with respect to the SM value of $J^{P}=0^{+}$, and the fraction of CP-odd contributions is measured to be $f_{a 3}=0.00_{-0.00}^{+0.23}$.

\section{References}

[1] S. Chatrchyan et al. [CMS Collaboration], Phys. Lett. B 716, 30 (2012)

[2] G. Aad et al. [ATLAS Collaboration], Phys. Lett. B 716, 1 (2012)

[3] Heinemeyer, S. et al. [LHC Higgs Cross Section Working Group] arXiv:1307.1347 (2013)

[4] Chatrchyan, S. et al. [CMS Collaboration], JINST 3, S08004 (2008)

[5] CMS collaboration, CMS Physics Analysis Summary HIG-13-002 (2013)

[6] S. Bolognesi et al., Phys. Rev. D 86095031 (2012)

[7] ATLAS and CMS Collaborations, LHC Higgs Combination Group, ATL-PHYS-PUB/CMS NOTE 2011-11, 2011/005, (2011). 